\title{
ANALISIS DEL DESARROLLO TEXTURAL DE CARBONES ACTIVADOS PREPARADOS A PARTIR DE ZURO DE MAÍZ
}

\section{ANALYSIS OF DEVELOPMENT TEXTURAL ACTIVATED CARBON FROM Zea mays}

\author{
Fredy Colpas", Arnulfo, A. Tarón², Waldyr Fong ${ }^{3}$
}

Recibido para publicación: Enero 28 de 2015 - Aceptado para publicación: Mayo 14 de 2015

\begin{abstract}
RESUMEN
Algunos residuos agrícolas pueden ser útiles para producir materiales adsorbentes como son los de carbones activados. Tal es el caso del Zuro de maíz, el cual fue utilizado como material de partida en esta investigación, Para la preparación de carbón activado, inicialmente el zuro libre de granos de maíz es carbonizado a temperatura de $400{ }^{\circ} \mathrm{C}$, posteriormente los carbonizados fueron activados químicamente con ácido ortofosfórico, los carbones activados obtenidos fueron modificados con ácido nítrico y peróxido de hidrógeno, previo calentamiento a $600^{\circ} \mathrm{C}$ para observar su desarrollo textural. Los sólidos fueron caracterizados a través de un análisis, para la determinación del área superficial se realizaron isotermas de adsorción mediante el método BET, utilizando equipo Micromeritics Gemini 2375. La activación con ácido ortofosfórico aumentó el contenido de carbono y disminuyó el contenido de oxígeno. El área de microporos se incrementó por el tratamiento con $\mathrm{HNO}_{3}$ y $\mathrm{H}_{2} \mathrm{O}_{2}$ desde $278,3 \mathrm{~m}^{2} \mathrm{~g}^{-1}$ hasta $401,7 \mathrm{~m}^{2} \mathrm{~g}^{-1}$ y $446,4 \mathrm{~m}^{2} \mathrm{~g}^{-1}$ respectivamente y la mayor parte del área total desarrollada correspondió a microporos. El pH generado por los carbones fue aproximadamente 3, el cual se determinó en soluciones acuosas luego de lavado con agua destilada; así mismo, estos materiales mostraron valores en los grupos $\mathrm{COOH}$ hasta 2,12 meq g-1.
\end{abstract}

Palabras Clave: Zuro de maiz, activación, ortofosfórico, microporos

\begin{abstract}
Some agricultural residues may be useful to produce adsorbent materials such as activated carbons. Such is the case of Zea mays, which was used as starting material in this research, this residue comes from the separation of the grains from the cob. For the preparation of activated carbon, initially free cob of corn kernels is carbonized at $400{ }^{\circ} \mathrm{C}$, subsequently carbonized were chemically activated with orthophosphoric acid, activated carbons obtained were modified with nitric acid and hydrogen peroxide preheating to $600{ }^{\circ} \mathrm{C}$ to observe the textural development. The solids were characterized by a final analysis for determining the surface

\footnotetext{
1"MSc. Ingeniería Química. Docente Titular. fcolpasc@unicartagena.edu.co. Móvil: 3126686031 Facultad de Ciencias Exactas y Naturales. Programa de Química. Universidad de Cartagena,Urb la Española Mza J Lote 11, Apto 201,Cartagena, Colombia.

${ }^{2}$ C.PhD. MSc. Biotecnología. Docente Titular. petetettaron@yahoo.com.mx Universidad de Cartagena, Urb los Jardines Mza F Lote 17, 1era Etapa, Cartagena, Colombia.

${ }^{3}$ MSc. Física. Director Programa de Metrología. Universidad de Cartagena. wfongs@unicartagena.edu.co, Barrio las Gaviotas Mza 23

Lote 2, 2da Etapa, Cartagena Colombia.
} 
area were conducted adsorption isotherms by the BET method using Micromeritics Gemini 2375. Activation with orthophosphoric acid increased carbon content and reduced oxygen content. Micropores area increased by treatment with $\mathrm{HNO}_{3}$ and $\mathrm{H}_{2} \mathrm{O}_{2}$ from $278.3 \mathrm{~m}^{2} \mathrm{~g}^{-1}$ hasta $401.7 \mathrm{~m}^{2} \mathrm{~g}^{-1}$ and $446.4 \mathrm{~m}^{2} \mathrm{~g}^{-1}$, respectively, most of the total developed area corresponded to micropores. The $\mathrm{pH}$ coals generated was approximately 3 , which was determined in aqueous solutions after washing with distilled water and these materials showed the same values in the $\mathrm{COOH}$ groups to $2.12 \mathrm{meq} \mathrm{g}^{-1}$.

Key word: Zea mays, activation, orthophosphoric, micropores

\section{INTRODUCCIÓN}

En Colombia y especialmente los departamentos de la costa caribe las materias primas de origen vegetal no son aprovechadas al máximo. Un ejemplo característico de esto sucede con el maíz, que solo se utiliza como materia prima para alimentos En la región Caribe se reportan grandes extensiones de tierra destinadas al cultivo de maíz en los municipios de Lorica, Cereté, San Carlos, San Pelayo, Montería, Cotorra, Chimá, San Carlos, Ciénaga de Oro, Tolú Viejo, Valencia, Fonseca, La Gloria, Sahagún, Villanueva, Corozal, Gamarra, San Martín, Montelíbano. La tuza o zuro de maíz, es un residuo resultante de la colecta del maíz cultivado, el cual con un contenido de lignina de $23,57 \%$, se convierte en material con un apreciable potencial para obtener carbones activados con el fin de remover metales pesados (Primera et al. 2011).

Colombia es uno de los países que tiene mayor diversidad de maíces nativos y criollos, que se expresa en las numerosas variedades y ecotipos de maíces presentes en las diferentes regiones y agroecosistemas del país, asociadas a las diversas expresiones culturales y socioeconómicas de las comunidades indígenas, afrocolombianas y campesinas. En la región caribe de Colombia, los cultivos de maíz ocupan los primeros lugares en cuanto a su producción agroindustrial, en el proceso de desgranado segenera un residuoconocidocomo zuro, el cual causa problemas ambientales por la falta de disposición final, trayendo como consecuencia impactos negativos al medio ambiente. La transformación de este residuo sin valor comercial y que además genera un impacto ambiental negativo, en un producto con valor comercial u objeto de investigación por la comunidad científica para encontrar alternativas de solución que a su vez ayuden al desarrollo económico y bienestar de los habitantes de la región. Los carbones activados son algunos de los materiales más usados a nivel industrial en trabajos de adsorción de gases, filtrados, limpieza de líquidos y como soporte para catalizadores en medios no oxidantes, estos materiales son sólidos obtenidos a partir del tratamiento químico o físico de materiales lignocelulosicos, cuyas características finales como área superficial, porosidad y grupos funcionales, dependen del precursor y del tipo de tratamiento empleado durante la síntesis. (Zhang 2010; Marsh1997).

Los carbones activados también son aplicados a la adsorción de contaminantes como gases e iones metálicos de plomo (Li 2010; Song 2010), cadmio y otros metales pesados (Garoma 2010; Kim 2011). La activación química normalmente se realiza a temperaturas inferiores a $900{ }^{\circ} \mathrm{C}$ en atmósfera inerte. Antes de este proceso, el sólido carbonáceo se impregna 
con una solución de agentes químicos que le confieren características que lo tornan útil para diferentes aplicaciones. A pesar de que existen muchas sustancias químicas que pueden conducir al proceso de activación, los más usados son el ácido fosfórico y el hidróxido de sodio, debido al bajo costo de estos y a las propiedades texturales y estructurales de los carbones producidos (Rodríguez-Reinoso y Molina-Sabio 1998). En la activación física se usan temperaturas parecidas a las usadas durante el proceso químico, pero en este segundo proceso la muestra carbonosa no se impregna con sustancias químicas y la atmosfera está constituida en la mayoría de los casos de dióxido de carbono (Navarrete et al. 2005).

Los carbones industriales son producidos a partir de sólidos carbonosos tales como, cascara de coco, zuro de maíz, cuesco de palma africana, carbón mineral, madera, también pueden ser usados como material de partida algas, cáscara de arroz, lignina, aserrín, cáscara de semilla de algodón, lignito, lodos de petróleo, semillas de frutas, melaza, carbón vegetal, desperdicios de pasta de papel, negro de humo, desechos de destilería, turba, carbohidratos, coque de petróleo, etc. (Burns 1999; Arpa 2000). Todos estos materiales poseen en común un alto contenido de carbono, y su bajo costo, característica que los hace viables para la obtención de carbón activado de acuerdo a lo planteado anteriormente.

El objetivo principal de esta investigación fue estudiar el potencial que tiene el zuro del Zea mays como materia prima para la obtención de carbones activados útiles en la remoción de contaminantes en fases acuosas permitiendo el aprovechamiento de residuos agrícolas generados en el desgranado del maíz.

\section{MATERIALES Y MÉTODOS}

En este trabajo se prepararon carbones activados a partir de zuro de maíz mediante carbonización a $400{ }^{\circ} \mathrm{C}$, activación con ácido fosfórico y oxidación con ácido nítrico o peróxido de hidrógeno seguido de tratamiento térmico, para analizar el desarrollo de las porosidad durante el proceso, teniendo en cuenta la composición química de los materiales en su superficie

\section{Carbonización del material de partida}

El material de partida (zuro de maiz) se colectó en la central de abastos de Bazurto (CartagenaColombia). El zuro fue pre-tratado a través de un proceso de secado a una temperatura de 105 ${ }^{\circ} \mathrm{C}$ durante 24 horas y posteriormente triturado. Para el proceso de carbonización, se utilizó $500 \mathrm{~g}$ de material seco en fracciones de 100 gramos, estas fueron calentadas en atmosfera de nitrógeno en un horno multipropósito Ohio Thermal Modelo VM 2 IC AI. ASTM - D 3174-97, desde la temperatura ambiente hasta los $400{ }^{\circ} \mathrm{C}$, con una rampa de calentamiento de $10{ }^{\circ} \mathrm{C} / \mathrm{min}$. El zuro se dejó en atmosfera de nitrógeno a $400^{\circ} \mathrm{C}$ durante $1 \mathrm{~h}$. Posteriormente el material carbonizado se retiró del equipo cuando la temperatura estuvo por debajo de $50{ }^{\circ} \mathrm{C}$. Luego de las carbonizaciones se determinó la pérdida de peso de las muestras teniendo en cuenta la masa; antes y después del proceso.

\section{Molienda y tamizado}

Los carbonizados obtenidos a partir del zuro de maíz se molieron en un molino helicoidal 
marca pulvex y se tamizaron a un tamaño de partícula de 125 a $300 \mu \mathrm{m}$ utilizando tamices Tyler estándar con malla número 50 y 120.

\section{Obtención de los carbones activados}

El carbón obtenido se impregnó con ácido ortofosfórico al $21 \% \mathrm{p} / \mathrm{v}$ con agitación constante por 4,5 h. Posteriormente la mezcla se filtró y el carbón resultante se secó a $105^{\circ} \mathrm{C}$ por 24 h. Seguidamente el carbón se calentó con una rampa de10 ${ }^{\circ} \mathrm{C} \min ^{-1}$ en atmósfera

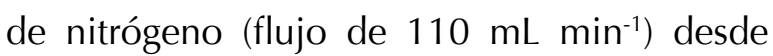
$30{ }^{\circ} \mathrm{C}$ hasta $450{ }^{\circ} \mathrm{C}$ y se mantuvo en esta condición por 120 minutos. (Tongpoothorn 2011) Finalmente el material activado fue lavado con agua caliente y fría hasta que la conductividad de las aguas de lavado fue muy similar a la conductividad del agua destilada, luego se secaron a $105^{\circ} \mathrm{C}$ durante 12 horas. (Huidobro y Rodríguez-Reinoso 2000). El material de partida se codificó como ZM y el carbón activado como ZM-A. Después de las activaciones se determinó la pérdida de peso de las muestras teniendo en cuenta el peso antes y después del proceso (Amalraj 2014).

\section{Modificaciones de los carbones activados Tratamiento térmico y oxidación}

ZM-A se usó como material de partida para la síntesis de dos nuevos materiales. Durante este proceso posterior, el ZM-A se pretrató térmicamente a $600{ }^{\circ} \mathrm{C}$ en atmósfera de nitrógeno $\left(110 \mathrm{~mL} \mathrm{~min}^{-1}\right)$ por $1 \mathrm{~h} \mathrm{y}$ seguidamente fue oxidado con ácido nítrico $\left(\mathrm{ZM}-\mathrm{HNO}_{3}\right)$ y peróxido de hidrógeno (ZM$\mathrm{H}_{2} \mathrm{O}_{2}$ ). Para el tratamiento con ácido nítrico, 20 g de ZM-A, se mezclaron con $250 \mathrm{~mL}$ de $\mathrm{HNO}_{3}$ al $15 \%$ por $0,5 \mathrm{~h}$; la mezcla se filtró, se lavó con agua destilada y se secó a $105^{\circ} \mathrm{C}$ por 24 h. Posteriormente el carbón activado seco se calentó en atmósfera de nitrógeno (Flujo de $110 \mathrm{~mL} \mathrm{~min}^{-1}$ ) desde $30{ }^{\circ} \mathrm{C}$ hasta $600{ }^{\circ} \mathrm{C}\left(10{ }^{\circ} \mathrm{C} \mathrm{min}^{-1}\right)$ y se mantuvo a estas condiciones por espacio de $1 \mathrm{~h}$. Para el tratamiento con peróxido de hidrogeno, $20 \mathrm{~g}$ de ZM-A se mezclaron con $250 \mathrm{~mL}$ de $\mathrm{H}_{2} \mathrm{O}_{2}$ al $10 \%$ por $0,5 \mathrm{~h}$; la mezcla se filtró, se lavó con agua destilada y se secó a $105{ }^{\circ} \mathrm{C}$ por 24 h; posteriormente, el sólido seco se calentó en atmósfera de nitrógeno (Flujo de $110 \mathrm{~mL}$ $\left.\mathrm{min}^{-1}\right)$ desde $30{ }^{\circ} \mathrm{C}$ hasta $600{ }^{\circ} \mathrm{C}\left(10^{\circ} \mathrm{C} \mathrm{min}^{-1}\right)$ y se mantuvo a estas condiciones por $1 \mathrm{~h}$.

\section{Caracterización de los Materiales}

Para la caracterización de los carbonizados, carbones activados y carbones activados modificados se practicaron los siguientes análisis: Análisis próximo, análisis último, pH de las suspensiones acuosas, análisis textural realizando isotermas de adsorción de nitrógeno y determinación de grupos oxigenados ácidos y básicos utilizando el método Boehnm (1994).

\section{Análisis próximo}

Mediante un análisis próximo se determinó el porcentaje de humedad en una estufa Precisión Scientific Modelo 18, materia volátil en un horno Ohio Thermal Modelo OCAF, cenizas y Carbono fijo por diferencia de todos los materiales obtenidos. En todos los análisis se utilizaron las normas ASTM 3302, ASTM 3175, ASTM 3174 y ASTM 3172.

\section{Análisis último}

ZM y ZM-A fueron caracterizados a través de análisis último usando la norma ASTM-D317889. En esta caracterización, la determinación de carbono e hidrógeno se realizó por pirolisis de los carbones en un sistema cerrado utilizando un Horno Lindbergh Modelo 123-4. ASTM 
- D 3178-89, determinando los productos de la combustión, dióxido de carbono y agua, después de la oxidación completa; el carbono y el hidrógeno se determinaron por el incremento de peso de trampas para $\mathrm{CO}_{2}$ y $\mathrm{H}_{2} \mathrm{O}$. En este proceso, el nitrógeno presente en los sólidos se convirtió en sales de amonio por digestión con ácido sulfúrico concentrado en una mezcla catalizada con sulfato de potasio a alta temperatura; las sales se disolvieron en una solución alcalina en caliente de la cual el amonio se evaporó y se determinó por titulación ácido-base colectándolo en un recipiente con una cantidad medida de ácido clorhídrico. Para la determinación de azufre total de los carbones se utilizó el método de la bomba lavadora SCFI (1982). En este método, los carbones se calcinaron a $800{ }^{\circ} \mathrm{C}$ por 1,5 $\mathrm{h}$ con una mezcla de óxido de magnesio y carbonato de bario que precipita el azufre como $\mathrm{BaSO}_{4}$ a partir del cual se calcula el contenido de este elemento. El porcentaje de oxígeno se calculó por diferencia. Estos resultados se corresponde a los reportados por Yu y colaboradores, donde realizan estudios de remoción con carbón activado oxidado con acido nítrico.

\section{Análisis textural de los materiales obtenidos}

Las medidas de área superficial específica y de microporosidad se obtuvieron a través de la adsorción de nitrógeno a $77 \mathrm{~K}$ por el método BET y por el t-plot, usando un equipo Micromeritics Gemini 2375. Previo a la adsorción del nitrógeno, las muestras fueron desgasificadas a $300{ }^{\circ} \mathrm{C}$ hasta una presión de $2 \mathrm{~mm}$ de $\mathrm{Hg}$. La adsorción se efectuó en el intervalo de valores de $\mathrm{p} / \mathrm{p}^{\circ}$ de 0,01 a 1 .

\section{Determinación de grupos oxigenados ácidos}

Para la determinación de los grupos carboxílicos se usó el método de Boehm (1994). En estos análisis se pesaron aproximadamente $0.5000 \mathrm{~g}$ de cada carbón y se agitaron a temperatura ambiente por 24 horas con 50 $\mathrm{mL}$ de una solución de $\mathrm{NaOH} \mathrm{0,1} \mathrm{N}$. Luego se filtró el carbón y la solución se tituló potenciométricamente con $\mathrm{HCl} 0,1 \mathrm{~N}$ para determinar la cantidad de reactivo consumido.

\section{Determinación de grupos oxigenados básicos}

Se pesaron aproximadamente 0,5 g de cada material y se colocaron en agitación constante a temperatura ambiente durante 24 horas con $50 \mathrm{~mL}$ de ácido clorhídrico $0,1 \mathrm{~N}$, luego se filtró y la solución se tituló con $\mathrm{NaOH} 0,1 \mathrm{~N}$ usando titulación potenciométrica (Boehnm 1994).

\section{Determinación del pH de los materiales obtenidos}

Para la determinación del $\mathrm{pH}$ de las soluciones acuosas generados por los carbones, se mezcló 1,0 g de cada carbón con 20 mL de agua destilada libre de $\mathrm{CO}_{2}$ en recipientes de vidrio y se agitaron a temperatura ambiente por 24 horas; luego, se midió el pH de las suspensiones acuosas con un electrodo de vidrio Orion 940 (Castilla et al. 2000).

\section{Análisis estadistico}

Todos los cálculos estadísticos se realizaron con la ayuda del programa statgraphics plus versión para Windows. Los resultados de acidez total, $\mathrm{pH}$, textura de los carbones se expresaron como promedio más o menos desviación estándar con el fin de determinar si existían diferencias significativas entre las áreas superficiales y las capacidades de adsorción 
Tabla 1. Resultados del Análisis Elemental del zuro de maíz original y activado con ácido ortofosfórico

\begin{tabular}{cccccc}
\hline Material & $\mathbf{C ~ ( \% )}$ & $\mathbf{H ~ ( \% )}$ & $\mathbf{N ~ ( \% )}$ & $\mathbf{S ~ ( \% )}$ & $\mathbf{O}(\%)$ \\
\hline ZM & $47,1 \pm 0,1$ & $6,9 \pm 0,1$ & $0,77 \pm 0,00$ & $0,09 \pm 0,00$ & $45,1 \pm 0,1$ \\
ZM-A & $74,0 \pm 0,2$ & $3,6 \pm 0,1$ & $0,57 \pm 0,08$ & $0,00 \pm 0,00$ & $21,8 \pm 0,02$ \\
\hline
\end{tabular}

de plomo y cadmio de todos los carbones se realizó test anova haciendo uso del programa estadístico STATGRAPHICS PLUS VERSIÓN para Windows.

\section{RESULTADOS Y DISCUSIÓN}

Durante la carbonización del zuro de maíz, las unidades de celulosa, principal componente de los materiales vegetales de partida, sufren depolimerización transformándose en unidades más pequeñas, se desprende materia volátil y se produce un sólido más rico en carbono.(Rodríguez-Reinoso y Molina-Sabio 1998).

En la tabla 1 se aprecian los resultados del análisis último del material vegetal de partida y del carbón activado con ácido ortofosfórico. El proceso de carbonización aumentó significativamente el contenido de carbono de ZM de 47 a 74\% (ZM-A) al tiempo que se disminuyó la cantidad de oxígeno e hidrógeno. Este aumento del contenido de carbono se debe al carácter deshidratante del ácido fosfórico, que facilita la pérdida de hidrógeno y oxígeno en forma de agua, proceso que ocurre simultáneamente con la compactación de unidades carbonadas grandes a unidades más pequeñas y enriquecidas en carbón. (Rodríguez- Reinoso y Molina-Sabio 1998; Mak 2009;).
El zuro de maíz está compuesto básicamente por lignina, celulosa y hemicelulosa; con la carbonización a $\quad 400{ }^{\circ} \mathrm{C}$, solo la celulosa y hemicelulosa se depolimerizan en unidades más pequeñas para dar origen a una gran masa rica en carbono, ya que la lignina se descompone a temperaturas mayores; sin embargo, la impregnación con el ácido fosfórico facilita la hidrólisis de la lignina que queda presente después del proceso de carbónizado, lo que conduce al aumento de propiedades texturales como el área superficial específica y la porosidad. (Berkowitz 1957; Kudo y Yoshida 1957).

Todos estos fenómenos de rompimiento de estructuras conducen de igual forma al aumento de la aromaticidad del carbón, la pérdida del carácter alifático y la formación de una estructura rígida entrecruzada. (Hsisheng 1998; Huidobro 2000). La tabla 2 muestra los valores de área superficial específica de los materiales activados. Se observa que en el carbón activado únicamente con ácido ortofosfórico, la mayor parte del área total es atribuida a la presencia de microporos, lo cual se debe al carácter deshidratante del mismo, el cual promueve la descomposición de grupos oxigenados principalmente. Esta descomposición estimula la formación de canales a través de los cuales el agua formada por la acción del ácido emerge del carbón, originando así la formación de los microporos.

(Wu y Zhao 2011). 
Tabla 2. Parámetros texturales, acidez de los carbones activados.

\begin{tabular}{|c|c|c|c|c|c|}
\hline Carbón & $\begin{array}{c}\text { Área de } \\
\text { microporos } \\
\left(\mathbf{m}^{2} / \mathbf{g}\right)\end{array}$ & $\begin{array}{l}\text { Área externa } \\
\left(\mathrm{m}^{2} / \mathrm{g}\right)\end{array}$ & $\begin{array}{l}\text { Área total } \\
\left(\mathrm{m}^{2} / \mathrm{g}\right)\end{array}$ & $\begin{array}{c}\text { Grupos COOH } \\
(\mathrm{meg} / \mathrm{g})\end{array}$ & $\begin{array}{c}\text { pH de } \\
\text { carbones }\end{array}$ \\
\hline ZM & $585,1 \pm 0,0004$ & $198,1 \pm 0,001$ & $778,26 \pm 0,007$ & $1,68 \pm 0,050$ & $5,6 \pm 0,2$ \\
\hline $\mathrm{ZM}-\mathrm{H}_{2} \mathrm{O}_{2}$ & $529,92 \pm 0,0010$ & $135,9 \pm 0,001$ & $663,78 \pm 0,003$ & $2,12 \pm 0,020$ & $5,3 \pm 0,1$ \\
\hline $\mathrm{ZM}-\mathrm{HNO}_{3}$ & $514,02 \pm 0,0020$ & $94,40 \pm 0,001$ & $606,40 \pm 0,003$ & $1,68 \pm 0,050$ & $5,1 \pm 0,1$ \\
\hline
\end{tabular}

En el caso de los carbones activados oxidados con ácido nítrico y peróxido de hidrogeno, la mayor parte del área superficial especifica total también se atribuye, en gran proporción, a los microporos presentes; sin embargo, estos carbones presentaron mayores áreas, lo cual se debe a que estos agentes químicos son oxidantes fuertes que conducen a la descomposición de parte de la estructura carbonácea a óxidos de carbono $\left(\mathrm{CO}_{2}\right.$ principalmente), originando nuevos poros que aumentan la microporosidad y el área total (Valix 2008).

El estudio de los grupos funcionales oxigenados presentes en las muestras activadas reveló que no existen grupos con carácter básico, es importante resaltar que la presencia de hidrogeno en la matriz del material se debe a su composición elemental tal como lo muestra el análisis último de la tabla1.

La tabla 2 muestra que el contenido de grupos $\mathrm{COOH}$ aumenta con la oxidación con peróxido de hidrogeno, mientras que el ácido nítrico no contribuye con este parámetro. $\mathrm{El} \mathrm{pH}$ de las suspensiones acuosas de los carbones es de carácter ácido (2,9 a 3,2), lo cual significa que estos carbones poseen una predominancia de grupos funcionales ácidos en su superficie, importantes para el proceso de adsorción de metales (López y Ramón 1999; Allen 2008).

\section{ANALISIS DEL DESARROLLO DEL AREA SUPERFICIAL}

En la figura 1 se observa las isotermas de absorción de los carbones activados: Las isotermas de adsorción de todos los materiales fueron tipo I (BET), igualmente el $70 \%$ del volumen de poros fue Ilenado a presiones relativas de 0,1 (p/p0) indicando que estas muestras son altamente microporosas (diámetro de poros menor a $20 \AA$; de Acuerdo a las gráficas de isoterma los carbones con mayor área superficial fue ZM-A.

\section{ESPESOR ESTADÍSTICO Y CURVA T PARA EL MÉTODO BET}

Cuando la adsorción de un gas sin condensación capilar ocurre sobre una superficie uniforme a una temperatura dada, se puede aceptar en primera aproximación, que el espesor de la capa adsorbida t, es función de la presión relativa.

$$
t=f(p / p o)
$$

Halsey, encontró que para el caso específico de adsorción de nitrógeno a $77 \mathrm{~K}$ el valor de $\mathrm{t}$ se podía obtener de la siguiente ecuación:

$$
t=-3,54(5 / \ln (p / p o))^{1 / 3}
$$




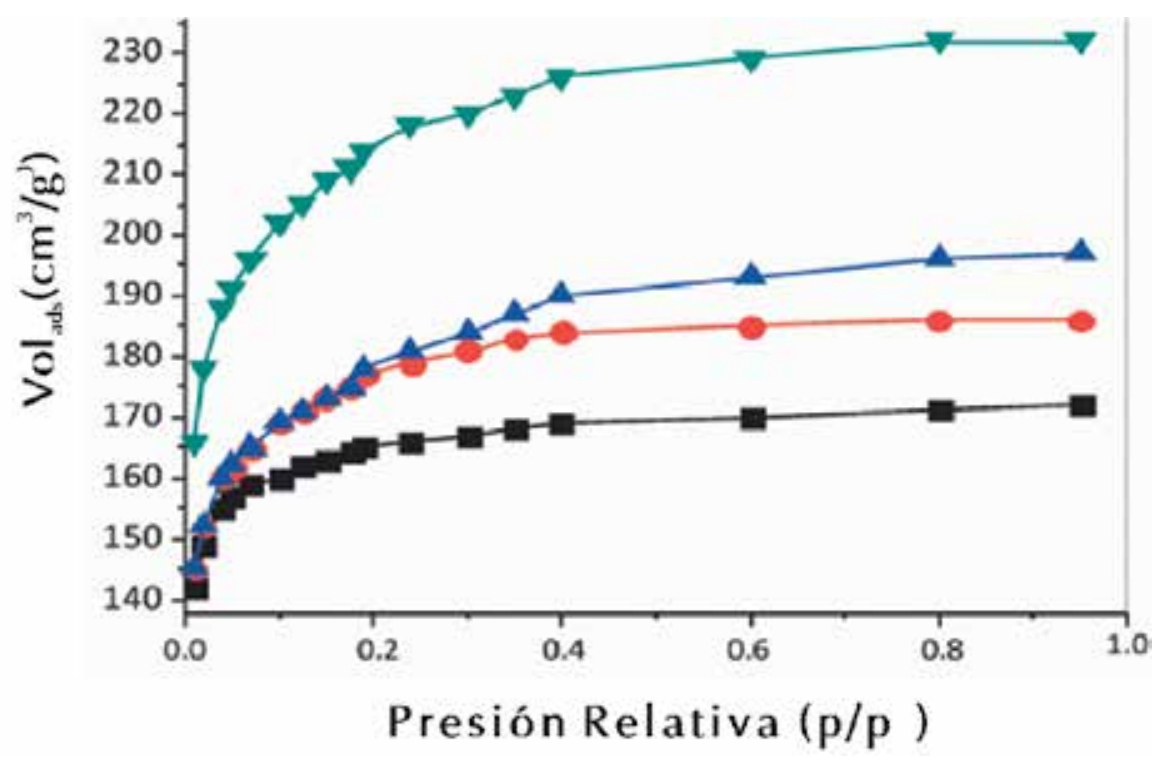

Figura 1. Isotermas de absorción tipo I (BET) de los carbones activados que indican que las muestras son microporosas: ZM-A $\boldsymbol{\nabla}, \mathrm{ZM}-\mathrm{H}_{2} \mathrm{O}_{2} \boldsymbol{\Delta}, \mathrm{ZMD}-, \mathrm{ZM}-\mathrm{HN}$.

Suponiendo que la estructura de poro no influye sobre la adsorción en multicapa y que la curva característica es válida, la representación gráfica del volumen adsorbido en función del espesor estadístico es una línea recta que pasa por el origen en el intervalo de presiones relativas donde no hay formación de multicapa. La pendiente de esta línea es proporcional al área específica del adsorbente. El área calculada a partir del espesor estadístico no siempre es igual al área calculada a partir del método BET. Sin embargo, cuando la relación (p/po) no produce desviaciones de la linealidad, el área calculada a partir de los valores de t es bastante próxima al área calculada a partir del método BET.

Para calcular el área de microporos, a partir de la relación del volumen adsorbido en función del espesor estadístico se determina el menor valor de la pendiente donde, y $(\varphi)$ calculamos el valor del área superficial externa $\left(A_{E x t}\right)$, y al restar éste valor del área obtenida por el método $B E T\left(A_{B E T}\right)$ Se obtiene el valor del área de microporos $\left(A_{M}\right)$.
$\mathrm{A}_{\text {Ext }}=10^{4} \varphi$, Donde $\varphi=$ pendiente entre $\mathrm{a} \mathrm{y} \mathrm{b}$

$A_{M}=A_{B E T}-A_{E x t}$

\section{CONCLUSIONES}

El zuro de maíz es un recurso agroindustrial potencial para la obtención de carbones activado microporosos. Se encontró que el proceso de activación aumentó significativamente el contenido de carbono del zuro de maíz, presentando un incremento de 47 a 74\% después de la activación.

Elaumento delárea ocasionadoporel tratamiento con ácido nítrico y peróxido de hidrogeno se debe al carácter oxidante de estas sustancias, las cuales descomponen parte de la estructura carbonosa, estimulando la formación de nuevos poros. Los carbones activados poseen una predominancia de grupos funcionales ácidos en su superficie, importantes para el proceso de 
adsorción de metales, especialmente aquellos que tengan un radio iónico proporcional al tamaño de los poros.

\section{REFERENCIAS}

Allen, S., Whitten, L. 2008. The production and characterization of activated coals: A Review. Asia-Pac. J. Chem. Eng. 6:231-261.

Amalraj, A., Pius, A. 2014. Removal of selected basic dyes using activated carbon from tannery wastes. Sep. Sci. Technol. 49: 90100.

Arpa, C., Basyilmaz, E., Bektas, S., Genc, O., \& Yurum, Y. 2000. Cation exchange properties of low Turkish coals: removal of $\mathrm{Hg}, \mathrm{Cd}$ and $\mathrm{Pb}$ from wastewater. Fuel Processing Technology 68(2):111-120.

Berkowitz, N. 1957. On the differential thermal analysis of coal. Fuel 36:355-373

Boehm, H.P. 1994. Some aspects of the surface chemistry of carbon blacks and other carbons. Carbon 32(5):759 -769.

Burns, C.A., Cass, J., Harding, I., Russell, H. 1999. Adsorption of aqueous heavy metals onto carbonaceous substrates. Colloids and Surfaces A155:63-68.

Castilla, M., López-Ramon, M.V., \& CarrascoMarin,F. 2000. Changes in surface chemistry of activated carbons by wet oxidation.Carbon 38(14):1995-2001.

Garoma, T., S., Shah, B., Badriyha. 2010. Removal of Cadmium, Copper, and Zinc from Aqueous Solution by Activated Carbon
Impregnated with 8-Hydroxyquinoline. J Environ Sci Eng 4(5):14-21.

González, J., Román, S., Encinar, J., Martínez, G. 2009. Pyrolysis of various biomass residues and char utilization for the production of activated carbons," J. Anal. Appl. Pyrolysis, 85(1-2):134-141.

Hsisheng, T., Yeh, T.S., \& Hsu, L-Y. 1998. Preparation of Activated Carbon from Bituminous Coal with Phosphoric Acid Activation. Carbon 36(9):1387-1395.

Huidobro, A., Pastor, A.C., \& Rodríguez-Reinoso, F. 2000. Preparation of activated carbon cloth from viscous rayon. Part IV. Chemical activation.Carbon 39(3):389-398.

Kim, D., Y. W., Jung, S., Kwon, J-W., Park. 2011. Adsorption of cadmium (II) from aqueous solutions by thiol-functionalized activated carbon. Water Sci. Technol.: Water Suppl. 11(1):61-66

Kudo,K.,\&Yoshida,E. 1957. Onthedecomposition process of wood constituents in the course of carbonization. Journal of the Japanese Wood Research Society 3 (4):125-127.

Li, Y., Q., Dua, X., Wang, P., Zhang, D., Wang, Z., Wang, Y., Xia. 2010. Removal of lead from aqueous solution by activated carbon prepared from Enteromorpha prolifera by zinc chloride activation. J Hazard Mat 183(1-3):583-589.

López-Ramón, M.V., Stoeckli, F., MorenoCastilla, C., \& Carrasco-Marín, F. 1999. On the characterization acidic and basic surface sites on carbons by various techniques. Carbon 37(8):12151221. 
Mak, S., Tey, B., Cheah, K., Siew, W., Tan, K. 2009. Porosity characteristics and pore developments of various particle sizes palm kernel shells activated carbon (PKSAC) and its potential applications. Adsorption 15: 507-519

Marsh, H., Heintz, E.A., \& Rodríguez-Reinoso, F. 1997. Introduction to Carbon Technology. Capítulo 2.Universidad de Alicante. Alicante, España: Secretariado de Publicaciones.

Navarrete, L., Giraldo, L., Baquero, M. 2005. Carbón activado: efecto del lavado con ácido sulfúrico del precursor lignocelulósico, cuesco de palma africana, sobre los procesos de carbonización y activación. Rev. Colombiana de Quím. 34:67-78.

Ospina, V., Buitriago, R., Lopez, L. 2014. Preparación y caracterización de carbón activado a partir de torta de higuerilla. Tecno Lógicas 17(32):75-84.

Primera, O., Colpas, F., Meza, E., Fernandez, R. 2011. Carbones activados a partir de bagazo de caña de azúcar y zuro de maíz para la adsorción de cadmio y plomo. Rev. acad. colomb. cienc. exact. fis. nat. 35(136): 387.396.

Rodríguez-Reinoso, F. \& Molina-Sabio,M. 1998. Carbones activados a partir de materiales lignocelulósicos. Química e Industria 45(9):563-571.

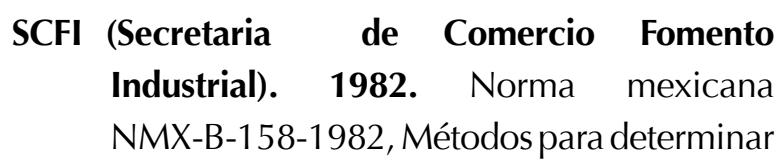

el azufre total en la muestra de carbón y coque. http://200.77.231.100/work/normas/ nmx/1982/nmx-b-158-1982.pdf.

Song, X., Liu, L., Chenga, \& Y., Qu. 2010. Surface modification of coconut-based activated carbon by liquid-phase oxidation and its effects on lead ion adsorption. Desalination 255(1- 3):78-83.

Tongpoothorn, W., Sriuttha, P., Homchan, S., Chanthai, C., Ruangviriyacha. 2011. Preparation of activated carbon derived from Jatropha curcas fruit shell by simple thermochemical activation and characterization of their physicochemical properties. Chem Eng Res Des 89(3):335340.

Valix, M., Cheung, W.H., Zhang, K. 2008. Textural and surface chemistry of activated bagasse and its role in the removal of chromium ions from solution. Adsorption 14:711-718.

Wu Z, Zhao D. 2011. Ordered mesoporous materials as adsorbents. Chem Commun; 47:3332-8.

Yu C, Fan X, Yu L, Bandosz TJ, Zhao Z, Qiu J. 2013. Adsorptive removal of thiophenic compounds from oils by activated carbon modified with concentrated nitric acid. Energy Fuel .27:1499-505.

Zhang, H., Yan, Y., Yang, L. 2010. Preparation of activated carbon from sawdust by zinc chloride activation.Adsorption 16:161166. 